\section{Principles and Practice of Clinical Research}

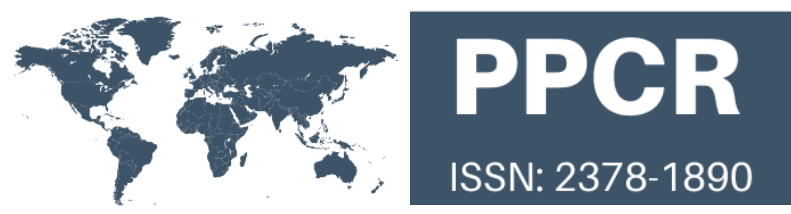

\title{
Protocol for a Phase I/II Trial to Evaluate Safety and Efficacy of Autologous Stem Cells Embedded in a Nanogel Patch for Patients with Heart Failure Undergoing Coronary Artery Bypass Graft
}

C. Vergara-Sanchez ${ }^{1}$, A. Dominguez-Rodriguez ${ }^{2}$, JA. Vasquez ${ }^{3}$, G. Grandez-Castillo4, AA. Elyas ${ }^{5}$, D. Hernandez-Herrero ${ }^{6}$, J. Eaton ${ }^{7}$, T. DuBois ${ }^{8}$, A. Schilling-Redlich ${ }^{9}$, C. Matsubayashi ${ }^{10}$, A. Paulino ${ }^{11}$, ACS. Teles ${ }^{12}$, E. Yamaguchi ${ }^{10}$, F. Gonzalez ${ }^{13}$, J. Camargo ${ }^{14}$, M. Nakai ${ }^{10}$, M. Aldosari ${ }^{15}$, O. BaptisteSainvil ${ }^{16}$, RA. Paes-Lima-Rocha ${ }^{17}$, R. Meneguetti ${ }^{18}$, S. Medeiros ${ }^{19}$, W. Yao ${ }^{20}$, WA. Mohamed ${ }^{21}$, I. Cosgarea $^{22}$, L. Candido-Santos ${ }^{23}$, Y. Chemale ${ }^{24}$, R. Nassir ${ }^{25}$, G. Castro-Tejada ${ }^{3}$, M. Toyama ${ }^{26}$, OF. Clerc $^{27}$, A. Sanchez-Jimenez ${ }^{28}$.

*Corresponding authors: Judith Vasquez, 3604 S W Young DR, Killeen, TX 76542, judith.vasquez.11@gmail.com

Rest of author's affiliation at the end of the manuscript.

Received April 23, 2020; accepted May 18, 2020; published May 19, 2020.

\begin{abstract}
:
Introduction: Heart failure affects numerous patients and carries a 50\% 5-year mortality rate from the time of diagnosis. Current treatment is based on medication, device therapy, revascularization and heart transplantation to reduce morbidity and mortality. However, stem cell therapy could improve cardiac function and outcomes, using a nanogel patch during coronary bypass graft.

Methods: An open label phase I/II trial consisting of an initial sentinel approach to test safety, followed by a randomized approach to test efficacy.

Discussion: Preclinical studies have shown safety of nanogel patches and clinical trials showed promise using mesenchymal stem cells. New approaches could contribute to the improvement of morbidity and mortality. Mesenchymal stem cell implantation with a nanogel patch could represent an effective and innovative therapeutic approach for patients with heart failure and reduced ejection fraction
\end{abstract}

Keywords: Mesenchymal Stem Cell Transplantation, Heart Failure, Coronary Artery Bypass, Research Design

DOI: http://dx.doi.org/10.21801/ppcrj.2020.61.4

\section{Abbreviations}

Congestive Heart Failure (CHF)

Angiotensin Converting Enzyme Inhibitors (ACEI)

Angiotensin II Receptor Blockers (ARB)

PerCutaneous Intervention (PCI)

Coronary Artery Bypass Graft (CABG)

Coronary Artery Disease (CAD)

Mesenchymal Stem Cells (MSC)

Surface Prone EpicArdial Delivery System (SPREADS)

Left Ventricular Ejection Fraction (LVEF)

Cardiac Magnetic Resonance Imaging (cMRI)

6-Minute Walk Test (6MWT).

N-Terminal Pro-Brain Natriuretic Peptide (NT-Pro-BNP test) Standard Of Care (SOC)

\section{INTRODUCTION}

Congestive heart failure (CHF) has been estimated to affect 6.2 million Americans with a prevalence expected to increase of $46 \%$ between 2012 and 2030 (Benjamin et al., 2019; Writing Group Members, 2016). From the time of diagnosis, patients have a 5-year mortality rate of approximately 50\% (Benjamin et al., 2019). Hospital admissions for heart failure in the United States generated annual costs exceeding 30 billion dollars in 2012 and are expected to increase to almost 70 billion dollars by 2030 (Benjamin et al., 2019). More than 50\% of patients with acute decompensated heart failure have a reduced ejection fraction (P. Chang et al., 2018). 
Coronary artery disease (CAD) is the most common risk factor for heart failure (Benjamin et al., 2019). Current standard of care(SOC) for heart failure is predominantly pharmacological, consisting of angiotensin converting enzyme inhibitors (ACEI), angiotensin II receptor blockers (ARB), angiotensin receptor-neprilisyn inhibitor (ARNI) or hydralazine and nitrate for African American population, plus a beta blocker and an aldosterone antagonist as indicated. Diuretics are used for volume overload (Yancy et al., 2017) (McMurray et al., 2014), newer therapies have been studied for specific populations like dapagliflozin which have also shown to decrease mortality they would be expected to be included soon in the guidelines (McMurray et al., 2019).

Several devices may be used in advanced heart failure like cardiac resynchronization therapy, inserted cardiac defibrillator or the left ventricular assisting device (Yancy et al., 2017). In patients with significant CAD, revascularization through percutaneous intervention (PCI) or coronary artery bypass graft (CABG) is indicated to improve myocardial perfusion, systolic function and outcome (Yancy et al., 2017), when patients with advanced CHF do not respond to optimal medical and resynchronization therapy there is indication for a heart transplantation (Alraies, M. C., \& Eckman, P. 2014). A novel trend is to use stem cells to help repair damaged cardiac tissue in heart failure patients with reduced ejection fraction. Although data are conflicting, autologous stem cells have been shown to be as safe and promising in human subjects (Beans, 2018) before considering transplantation.

Bone marrow is composed of numerous undifferentiated stem cells. One subtype is mesenchymal stem cells (MSC). In animal models, MSCs were shown to improve cardiac function when injected into injured myocardial tissue. (Duelen \& Sampaolesi, 2017). They use paracrine signaling to communicate with, direct, and modulate the cellular microenvironment promoting survival and proliferation of endogenous cells. This signaling further induces angiogenesis, quells inflammation, inhibits apoptosis, and recruits endogenous progenitor cells to endpoint differentiation, ultimately leading to improvement in contractile function (Mingliang et al., 2011). MSCs are also able to secrete potent levels of interleukin-1 receptor antagonist, which may play a key role as a regulator in MSCs-based therapy. (Duelen \& Sampaolesi, 2017)

In a preclinical study, a minimally invasive fluid hydrogel epicardial patch, known as Surface Prone EpicArdial Delivery System (SPREADS), was a safe method for carrying autologous bone marrow MSCs.
Cardiac function was significantly improved by regional administration of SPREADS with cells encapsulated in a hyaluronic acid-based hydrogel, demonstrated by an increase in left ventricular ejection fraction (LVEF) (Dolan et al., 2019). Additionally, nanogel matrix, a type of hydrogel in nanoscale, has demonstrated a cell retention 4 times higher compared to stem cells alone, with improvements in heart function, increased angiogenesis, and decreased fibrosis (Marquardt \& Heilshorn, 2016; Xia et al., 2015).

Some clinical trials using bone marrow-derived stem cell therapies in human patients with CAD and CHF were found to decrease long term mortality and to increase left ventricular ejection fraction. However, methods vary in terms of placement and source, leading to diverse results (Behbahan et al., 2015; Fisher et al., 2016). Other clinical trials using autologous stem cell applications in humans are inconclusive regarding cardiac regenerative benefits but are suggestive of safety. (Banerjee et al., 2018; Beans, 2018). Some of the probable adverse effects detected of the nanogel patch use are increased risk of arrhythmia (Beans 2018), immunemediated inflammatory reaction, increased risk of neoplasia and death (Fisher 2014, Xia 2015).

Several studies have shown positive effects in left ventricular function using MSCs in cardiac applications, but there is controversy with the results, mainly because MSCs alone have poor survival rates in the tissue. Additional preclinical studies used patches to overcome this poor cell survival and improve results (Dolan et al., 2019). However, no clinical trial has ever assessed the safety and efficacy of a nanogel patch embedded with autologous MSCs in humans. This study is intended to be a proof of concept. We propose the implantation of a nanogel patch embedded with autologous MSCs at the time of CABG in patients with HF with reduced LVEF and NYHA II-III referred to myocardial revascularization, to support cell regeneration and improve cardiac function in a 1-year follow-up period.

\section{Objectives}

\section{Primary objectives}

- Efficacy of nanogel patches with MSCs to improve LVEF measured by cardiac Magnetic Resonance Imaging (cMRI), after a one-year follow-up.

- Serious adverse events (SAEs): Cardiovascular and procedure-related mortality, lifethreatening ventricular tachycardia and ventricular fibrillation, Acute Myocardial Infarction (AMI) and hospitalization due to cardiovascular events. 
Secondary objectives

Efficacy and safety of our intervention by monitoring:

- NYHA Score improvement

- 6-minute walk test (6MWT).

- NT-Pro-Brain Natriuretic Peptide (NT-Pro-BNP)

\section{MATERIALS AND METHODS}

\section{Trial design}

This will be an open-label, interventional, controlled, randomized, single-center, phase I/II exploratory trial. It will begin with a sentinel approach in 9 patients as a runin phase. The 3 first patients will receive half the dose of nanogel patch with stem cells during CABG. If no complications occur in the following 3 months, then the other 6 patients will receive a full dose of the intervention (Figure 1). If no complications occur within 6 months, the study will continue to phase II, where the intervention group will receive the nanogel patch with MSCs during $\mathrm{CABG}$, whereas the control group will undergo usual CABG, followed by Standard Of Care (SOC) in both groups. The study will be stopped if any patient develops a SAE attributable to the intervention. The protocol will be submitted for Institutional Review Board approval prior to initiating the study.

\section{Statistical analysis for primary and secondary outcomes, sample size calculation and missing data}

For the primary outcome, we will use an unpaired t-test. SAEs will be analyzed as time-to-event through Kaplan-
Meier curves and log-rank tests along with hazard ratio. Frequency of SAEs will be compared between groups using chi square. Medians, interquartile ranges and 2sided p-values will be reported using an alpha $\leq 0.05$ as level of significance. For missing data, we will implement multiple imputation. STATA 16 will be used for the statistical analysis.

Sample size was calculated with an alpha of 0.05 , a power of $80 \%$ and a delta in LVEF of 4.05 , according to a meta-analysis (Fischer 2016). The estimated dropout rate was $10 \%$. The final sample size was 62 patients.

\section{Randomization and allocation concealment}

After giving informed consent, patients will be randomized during the second part of the phase I/II trial using single block randomization with a 1:1 allocation ratio adjusted by age and LVEF using the ALEA clinical tool (https://www.aleaclinical.eu/).

\section{Blinding}

To evaluate patient safety, this study will be open label, in agreement with FDA guidance for early-phase trials with cellular and gene therapy products (https://www.fda.gov/media/106369/download).

Thus, surgeons, treating physicians and their staff will not be blinded. However, data analysts, outcome assessors, radiologists interpreting $\mathrm{cMRI}$, cardiologists interpreting the echocardiograms, and the staff performing the periodic follow-up phone calls will all be blinded to group allocation.

\section{STUDY PLAN \\ Phase I}

\section{SENTINEL APPROACH}

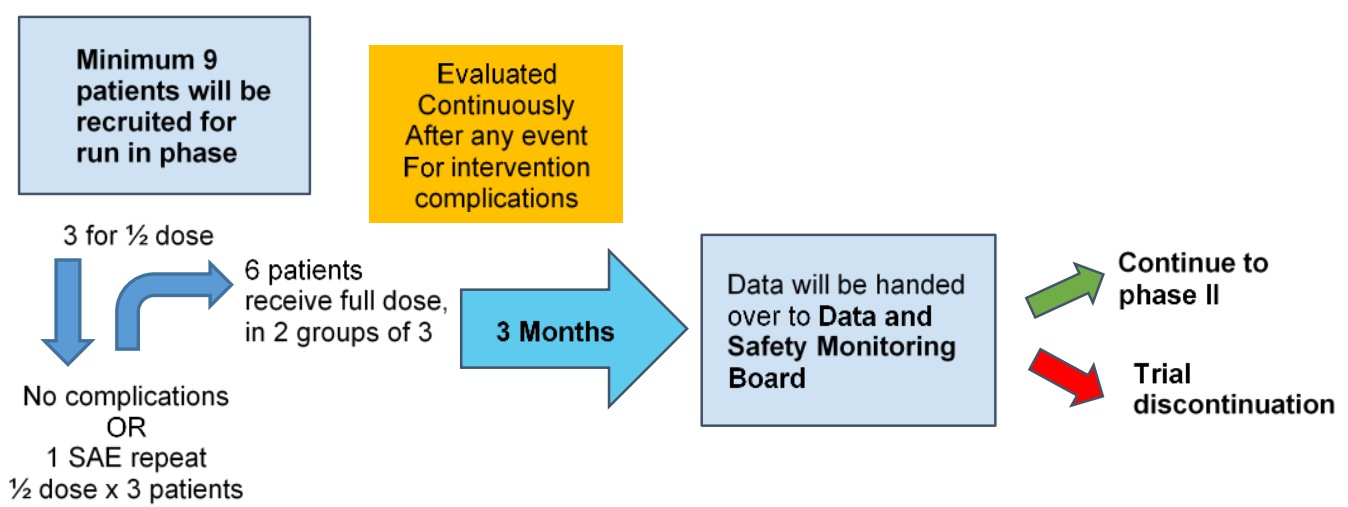

Figure 1. Trial Design. Description of the phase I/II with a sentinel approach to test for safety in a run-in phase before continuing to test efficacy. SAE: Serious Adverse Events. 


\section{Eligibility Criteria}

\section{Inclusion Criteria}

1. Men and women, age 40 to 75 years old.

2. Revascularization by CABG indicated by heart team decision, based on AHA guidelines 2011 criteria.

3. Hemodynamic stability based on shock index parameters.

4. LVEF $30-50 \%$ as assessed by echocardiography or cMRI.

5. CAD as the likely cause of heart failure as per patient's history and cardiac workup evaluation.

6. NYHA class II-III.

7. On optimal medical treatment as defined for the specific patient class and stage according to the 2017 update of the AHA guidelines for the management of heart failure and using new medical therapy approved by the FDA according to individual assessment and patient profile in the light of current medical practice.

\section{Exclusion Criteria}

1. Any cognitive condition that prevents the subject from understanding the nature of the protocol and granting his/her consent.

2. Pregnant or breastfeeding women, hormonal replacement therapy or combined contraceptive therapy.

3. Participation in another clinical trial within 30 days prior to inclusion.

4. Life expectancy of less than 1 year for any reason.

5. Stroke within 12 months prior to inclusion or with disability from prior stroke.

6. Society of Thoracic Surgeons score for mortality $>8 \%$.

7. Moderate-severe chronic obstructive pulmonary disease (COPD GOLD >III).

8. Glomerular filtration rate $<30 \mathrm{~mL} / \mathrm{min} / 1.73 \mathrm{~m} 2$.

9. Prior cardiac surgery or thorax radiation.

10. Heavily calcified proximal aorta or poor target vessels for CABG.

11. Indication to $\mathrm{CABG}$ plus other cardiac procedures, such as valve replacement/repair.

12. Inability to perform a 6-minute walk test.

13. An episode of significant arrhythmia in the last 6 months.

14. Bone marrow dysfunction.

15. Prior treatment with cell or gene therapy.

16. Cancer in the last 12 months.

17. History of autoimmune disease.

18. History of primary or acquired immunodeficiency or on immunosuppressive therapy.
19. Current smoking or cessation for less than 6 months.

20. Uncontrolled diabetes mellitus (HbA1c: $>8,5 \%$ ).

21. Active infection with White Blood Cell count $>13.0 \times 10^{3} / \mathrm{uL}$ or C Reactive Protein $>10 \mathrm{mg} / \mathrm{dl}$ or recorded fever (temperature $>37.8^{\circ} \mathrm{C}$ ) in the last 30 days.

22. Active alcohol dependence and/or recreational illegal drug use.

23. Moderate to severe valvular disease (stenosis or insufficiency).

24. Major congenital or acquired cardiovascular malformations.

25. Patients whose nanogel patch cannot be manufactured.

26. History of allergy or hypersensitivity to the components of the nanogel patch.

27. Any condition assessed by the heart team as being inappropriate for the study.

\section{Recruitment Strategy}

Target awareness campaigns will take place at local meetings of cardiology-related specialties and through websites and social media of heart failure patients associations, an official website of the trial with the main information and the contact of the research team will be available.

Interested patients will meet the study staff, who will review the protocol and available clinical information with the patient. After the informed consent is signed, the eligibility assessments will be performed. Patients fulfilling the inclusion criteria will be re-evaluated to reconsent before the procedure. A disclosure agreement will also be completed for medical record review in the case of participant withdrawal from the protocol, but willing to give information from other sources about outcomes.

\section{Adherence}

Adherence will be encouraged and monitored to maintain study compliance as high as possible. The main strategy will consist of open communication between the patient and the study team, supported by constant awareness of the study, and psychological care to the patient and family.

Patient and family will self-report symptoms and measurements like EKO device monitoring (a portable handheld telemonitoring device with ECG and electronic stethoscope technology https://www.ekohealth.com/). Telephone and home visits will be performed between the office visits, and a 24/7 phone line to reach a member of the research team will be available to participants. 
Adherence to medication regimen will be improved using single tablets with combined medications and once daily dosages when applicable. Costs of optimal heart failure pharmacological treatment and rehabilitation not covered by the insurance will be provided during the trial, as well as transportation expenses and the EKO monitoring. Patients will be able to discontinue their participation in the study at any time. There will be no change in surgical schedule for patients withdrawing prior to CABG.

\begin{tabular}{|c|c|c|}
\hline DAY 0 & \multirow[t]{2}{*}{ DETECTION } & DETECTION \\
\hline Day $1( \pm 7 \mathrm{~d})$ & & PRE-PROCEDURE VISIT 1 \\
\hline \multirow[t]{2}{*}{$\begin{array}{l}\text { Day } 7( \pm 3 \mathrm{~d}) \\
\text { Day } 10( \pm 3 \mathrm{~d})\end{array}$} & \multirow[t]{3}{*}{$\begin{array}{l}\text { PRE-PROCEDURE } \\
\text { ENROLLMENT }\end{array}$} & \\
\hline & & PRE-PROCEDURE VISIT 3 \\
\hline Day $15( \pm 7 d)$ & & $\begin{array}{l}\text { PRE-PROCEDURE VISIT } 4 \\
\text { Bone Marrow Extraction }\end{array}$ \\
\hline \multirow{3}{*}{$\begin{array}{c}\text { Day } 30( \pm 7 \mathrm{~d}) \\
0( \pm 1 \mathrm{~d}) \\
\text { Week } 1( \pm 3 \mathrm{~d})\end{array}$} & BASELINE & \multirow{3}{*}{$\begin{array}{l}\text { ADMISSION } \\
\text { PROCEDURE } \\
\text { DISCHARGE }\end{array}$} \\
\hline & & \\
\hline & PROCEDURE & \\
\hline TIME & \multirow[t]{10}{*}{ FOLLOW UP } & TYPE OF VISIT \\
\hline Week $2( \pm 2 \mathrm{~d})$ & & HOME FOLLOW UP \\
\hline Week $3( \pm 2 \mathrm{~d})$ & & PHONE FOLLOW UP \\
\hline & & \\
\hline 1 month $( \pm 5 d)$ & & OFFICE VISIT \\
\hline 2 months $( \pm 2 \mathrm{~d})$ & & $\begin{array}{l}\text { PHYSICAL THERAPY } \\
\text { FOLLOW UP }\end{array}$ \\
\hline 3 months $( \pm 5 \mathrm{~d})$ & & OFFICE VISIT \\
\hline 4.5 months $( \pm 2 \mathrm{~d})$ & & PHONE FOLLOW UP \\
\hline $\begin{array}{l}6 \text { months }( \pm 5 \mathrm{~d}) \\
7.5 \text { months }( \pm 2 \mathrm{~d}) \\
9 \text { months }( \pm 7 \mathrm{~d})\end{array}$ & & $\begin{array}{c}\text { OFFICE VISIT } \\
\text { PHONE FOLLOW UP } \\
\text { HOME VISIT }\end{array}$ \\
\hline $\begin{array}{c}10.5 \text { months }( \pm 2 \mathrm{~d}) \\
12 \text { months }( \pm 7 \mathrm{~d})\end{array}$ & & $\begin{array}{l}\text { PHONE FOLLOW UP } \\
\text { OFFICE VISIT }\end{array}$ \\
\hline
\end{tabular}

Table 1. Timeline of follow up for each patient in the study.

\section{Procedures}

\section{Stem Cell Harvesting}

Bone marrow aspiration will be performed in consented patients allocated to the intervention group. Under local anesthesia, 50 to $60 \mathrm{~mL}$ of bone marrow will be collected from the iliac crest according to standards described by
Chahla (Chahla et al., 2017). The sample will be screened and immediately transported to a governmentally regulated product manufacturing facility to be processed according to the protocol published by Akimoto (Akimoto et al., 2018) and the American Society of Transplantation and Cellular Therapy.

Synthesis of the MSC embedded nanogel patch

The cultured MSCs will be suspended within a thermosensitive nanogel. The nanogel is composed of poly (N-isopropylacrylamide-co-acrylic acid) (P(NIPAM$\mathrm{AA})$ ), a liquid solution under $30^{\circ} \mathrm{C}$ that changes into a gel at $37^{\circ} \mathrm{C}$. The nanogel is synthesized by polymerization according to the protocol described by Tang J et al. (Tang et al., 2017). The gel and MSCs are mixed to achieve a result of $1 \times 105$ cells in $125 \mu \mathrm{L}$ of P(NIPAM-AA) nanogel. The encapsulation of MSC will be completed as described in Tang J et al. (Tang et al., 2017)

Injection of stem cells

After the sternotomy for CABG, the cooled nanogel solution embedded with autologous MSCs is injected into the myocardium of the left ventricular wall near the site of revascularization for improved perfusion to the surrounding cardiac tissue, aiming at a greater potential for stem cell survival. Placement of the nanogel will be performed using a robotic injection device with temperature control configuration developed by Zhu et al. operated through virtual image guidance, displayed to the surgeon on a computer control screen. Therefore, the risk of myocardial damage is minimized. After injection, the solution transforms into a gel at $37^{\circ} \mathrm{C}$ for adherence to the myocardial tissue. (Zhu et al., 2016)

\section{Modification/discontinuation: Dose escalation}

The medical evaluations and patient-reported outcomes will be compared to previous cardiac exams and documentation provided by the patient, in order to proactively detect the impairment of cardiac function. If impaired cardiac function or other adverse events are detected, they will be reported promptly to the Safety Data Monitoring Committee (SDMC) and discussed between the investigators, the patient's primary cardiologist, and the patient to determine the most appropriate course of action.

The categorization of adverse events will be based on the following parameters (FDA 2018):

1. Seriousness, defined as negative outcomes such as death or prolonged hospitalization period, related to the nanogel patch embedded with autologous MSCs, or the necessity of medical or surgical intervention to prevent the negative outcomes related above. 
2. Expectedness of an event. An event will be considered unexpected if it has not been previously observed, documented, or considered as a possible event prior to the application of the patch, but the event occurred after the intervention and during the trial.

3. Relatedness, in reference to the likelihood of establishing a degree of causality to the exposure of our intervention.

\section{Data Management and monitoring}

We will use electronic case report forms to manage data through the cloud based software, CastorEDC (https://www.castoredc.com/), for electronic data capture (EDC) to create, maintain, retrieve, transmit, and monitor data. All data collection will be standardized through entry into the cloud program by study personnel directly from medical devices, ensuring personal and timestamped inputs according to specific user roles to protect the integrity and uniformity of the data, that will be monitored and recorded within the secure and encrypted cloud software.

An independent safety SDMC will be appointed to monitor the trial, ensuring trial progress and safety of patients. The monitoring committee will consist of a statistician and 3 cardiologists experienced in clinical trial procedure, development, and practice, but not directly involved in the study or with competing interests.

\section{DISCUSSION}

CAD and consequent CHF are leading causes of morbidity and mortality around the world. Despite significant medical advances in medication, device therapy, and revascularization techniques, 5-year mortality rate remains elevated (Benjamin et al., 2019). Regenerative therapies, that have been in development for more than 10 years, have had limited clinical impact, but have given a strong background to better understand mechanisms of myocardial repair at the molecular level, and constitute a promising therapeutic approach, motivating this trial.

Preclinical evidence showed the safety of mesenchymal stem cells injected into the myocardial tissue. But their limited ability to grow in a necrotic environment limited the clinical impact of this approach. Thus, regenerative initiatives like the one we propose from Zhu are focused on improving the method of cell delivery to provide a stimulating environment supporting controlled growth and orienting the differentiation of the regenerated tissue.

As these specific patch, technique, and dose of cells have never been tested together in humans, we propose a unique phase I/II sentinel approach to guarantee a closer observation and to limit the exposure to adverse events and unnecessary interventions. Moreover, our protocol enables a safe and rapid progression to phase II with assessment of clinical impact at 12 months, following the recommendations of FDA for the design of early-phase clinical trials of cellular and gene therapy products, given that safety of each component alone has been demonstrated.

\section{Limitations}

Recruitment of patients for a first-in-human trial always constitutes an ethical and practical challenge. Patients should fulfill requirements to justify the risk of the procedure, while being in the best possible physical and psychological conditions to tolerate the additional intervention, and the rigor of the protocol and prevent a high dropout rate.

We assessed this limitation by proposing a target awareness recruitment strategy combined with a strong and large group of highly trained and dedicated physicians in a multidisciplinary heart team that will perform a close and constant follow-up, complemented with patient and family inclusion, as well as telemonitoring.

Not blinding participants or physicians could affect not subjective outcomes like the NYHA score improvement, nevertheless, external blinded outcome assessors are used for this and most outcomes are objective in nature to overcome this. Finally, the learning curve of the procedure using the robotic assisted injection device could induce a potentially deleterious prolongation of the on-pump time, particularly for patients with lower LVEF. Therefore, pre-procedure training in animal models will be implemented.

\section{Impact of the Study}

Research with MSCs in CHF has shown controversial results throughout the years, which may be due to the various approaches used to deliver the cells into the myocardium. However, there is a biological plausibility of the effectiveness of stem cells to repair diseased hearts. Thus, to achieve efficacy in reversing heart damage with stem cells, we want to explore a new way of administering them to treat a disease with high morbidity and mortality worldwide. We believe that a nanogel patch can prove to be safe and effective for stem cell delivery into the myocardium, based on preclinical studies showing some success. As technology moves forward, patient care should move along the same way, and regenerative medicine is a very promising horizon to explore for this purpose. 


\section{Acknowledgements}

We are grateful to Professor Felipe Fregni and all the PPCR team (teaching assistants, faculty, coordinators) for their help during the design and review of this manuscript and the colleagues from the Team Humanitas for their help.

\section{Author Affiliations}

1 Pontificia Universidad Javeriana Cali, Colombia

2 Angiografía de Occidente Cali Colombia

3 Pontificia Universidad Catolica Madre y Maestra, Santiago,

Dominican Republic

4 University of San Martin de Porres, Lima, Peru.

5 Hamad Medical Corporation, Doha, Qatar

6 Hospital Universitario La Paz. Madrid, Spain

7 Institute for Family Health, New York, USA

8 Nova Biomedical Inc. Waltham, MA, USA

9 Facultad de Medicina Clinica Alemana -Universidad del Desarrollo, Chile

10 University of Sao Paulo, Sao Paulo Brazil

11 Ministerio de Salud Pública, Santo Domingo, Dominican Republic

12 Hospital Sirio Libanes, Brasil.

13 Hospital da Força Aérea do Galeão/ IBTS Rio de Janeiro, Brazil 14 Instituto de Oncología do Paraná, Brasil

15 Primary health care, Doha, Qatar

16 Hospital Metropolitano de Santiago, Dominican Republic

17 Universidade la Salle, Brazil

18 Instituto Brasileiro de Controle do Câncer - IBCC, Brasil.

19 Hospital Evangelico, Belo Horizonte, Brazil

20 Palleon Pharmaceuticals, Boston USA

21 Hamad Medical Corporation, Doha, Qatar

22 Translational and Clinical Research Institute, Newcastle University Center for Cancer

23 Massachusetts General Hospital, Harvard Medical School, Boston USA

24 Hospital Municipal e Maternidade Ruth Cardoso - Deltamed, Brasil

25 Department of Pathology, Faculty of Medicine, Umm Al-Qura University, Mecca, Saudi Arabia

26 Kyoto University, Graduate School of Medicine, Kyoto Japan

27 University Hospital Basel, Switzerland

28 T. H. Chan Harvard School of Public Health, Boston USA.

\section{Conflict of interests}

The authors declare no conflict of interests.

\section{REFERENCES}

Akimoto, A., Niitsu, E., Nagase, K., Okano, T., Kanazawa, H., \& Yoshida, R. (2018). Mesenchylmal Stem Cell Culture on Poly(N-isopropylacrylamide) Hydrogel with Repeated Thermo-Stimulation. In International Journal of Molecular Sciences (Vol. 19, Issue 4, p. 1253). https://doi.org/10.3390/ijms19041253

Alraies, M. C., \& Eckman, P. (2014). Adult heart transplant: indications and outcomes. Journal of thoracic disease, 6(8), 1120-1128. https://doi.org/10.3978/j.issn.2072-1439.2014.06.44

Banerjee, M. N., Bolli, R., \& Hare, J. M. (2018). Clinical Studies of Cell Therapy in Cardiovascular Medicine. In Circulation Research (Vol. 123, Issue 2, pp. 266-287). https://doi.org/10.1161/circresaha.118.311217
Beans, C. (2018). Inner Workings: The race to patch the human heart. Proceedings of the National Academy of Sciences of the United States of America, 115(26), 6518-6520.

Behbahan, I. S., Keating, A., \& Gale, R. P. (2015). Bone Marrow Therapies for Chronic Heart Disease. In STEM CELLS (Vol. 33, Issue 11, pp. 3212-3227). https://doi.org/10.1002/stem.2080

Benjamin, E. J., Muntner, P., Alonso, A., Bittencourt, M. S., Callaway, C. W., Carson, A. P., Chamberlain, A. M., Chang, A. R., Cheng, S., Das, S. R., Delling, F. N., Djousse, L., Elkind, M. S. V., Ferguson, J. F., Fornage, M., Jordan, L. C., Khan, S. S., Kissela, B. M., Knutson, K. L., ... American Heart Association Council on Epidemiology and Prevention Statistics Committee and Stroke Statistics Subcommittee. (2019). Heart Disease and Stroke Statistics-2019 Update: A Report From the American Heart Association. Circulation, 139(10), e56-e528.

Chahla, J., Mannava, S., Cinque, M. E., Geeslin, A. G., Codina, D., \& LaPrade, R. F. (2017). Bone Marrow Aspirate Concentrate Harvesting and Processing Technique. Arthroscopy Techniques, 6(2), e441-e445.

Dolan, E. B., Hofmann, B., de Vaal, M. H., Bellavia, G., Straino, S., Kovarova, L., Pravda, M., Velebny, V., Daro, D., Braun, N., Monahan, D. S., Levey, R. E., O'Neill, H., Hinderer, S., Greensmith, R., Monaghan, M. G., SchenkeLayland, K., Dockery, P., Murphy, B. P., ... Duffy, G. P. (2019). A bioresorbable biomaterial carrier and passive stabilization device to improve heart function post-myocardial infarction. Materials Science \& Engineering. C, Materials for Biological Applications, 103, 109751.

Duelen, R., \& Sampaolesi, M. (2017). Stem Cell Technology in Cardiac Regeneration: A Pluripotent Stem Cell Promise. EBioMedicine, 16, 3040.

E6(R2) Good Clinical Practice: Integrated Addendum to ICH E6(R1) Guidance for Industry. U.S. Department of Health and Human Services Food and Drug Administration (FDA). March 2018. https://www.fda.gov/media/93884/download. Accessed 20 Apr 2020.

Fisher, S. A., Doree, C., Mathur, A., Taggart, D. P., \& Martin-Rendon, E. (2016). Stem cell therapy for chronic ischaemic heart disease and congestive heart failure. Cochrane Database of Systematic Reviews , 12, CD007888.

Gersh BJ, Maron BJ, Bonow RO, et al. 2011 ACCF/AHA guideline for the diagnosis and treatment of hypertrophic cardiomyopathy: a report of the American College of Cardiology Foundation/American Heart Association Task Force on Practice Guidelines. J Am Coll Cardiol. 2011;58: e000-000.

Marquardt, L. M., \& Heilshorn, S. C. (2016). Design of Injectable Materials to Improve Stem Cell Transplantation. Current Stem Cell Reports, 2(3), 207220.

Mcmurray, J. J., Packer, M., Desai, A. S., Gong, J., Lefkowitz, M. P., Rizkala, A. R., ... Zile, M. R. (2014). Angiotensin-Neprilysin Inhibition versus Enalapril in Heart Failure. New England Journal of Medicine, 371(11), 993-1004. doi: 10.1056/nejmoa1409077

Mcmurray, J. J., Solomon, S. D., Inzucchi, S. E., Køber, L., Kosiborod, M. N., Martinez, F. A., ... Langkilde, A.-M. (2019). Dapagliflozin in Patients with Heart Failure and Reduced Ejection Fraction. New England Journal of Medicine, 381(21), 1995-2008. doi: 10.1056/nejmoa1911303

Mingliang, R., Bo, Z., \& Zhengguo, W. (2011). Stem cells for cardiac repair: status, mechanisms, and new strategies. Stem Cells International, 2011, 310928.

Patricia P. Chang , Lisa M. Wruck, Eyal Shahar, Joseph S. Rossi, Laura R. Loehr, Stuart D. Russell, Sunil K. Agarwal, Suma H. Konety, Carlos J. Rodriguez and Wayne D. Rosamond (2018). Trends in Hospitalizations and Survival of Acute Decompensated Heart Failure in Four US Communities (20052014). Circulation. 2018;138:12-24. https://doi.org/10.1161/CIRCULATIONAHA.117.027551.

Tang, J., Cui, X., Caranasos, T. G., Hensley, M. T., Vandergriff, A. C., Hartanto, Y., Shen, D., Zhang, H., Zhang, J., \& Cheng, K. (2017). Heart Repair Using Nanogel-Encapsulated Human Cardiac Stem Cells in Mice and Pigs with Myocardial Infarction. ACS Nano, 11(10), 9738-9749.

Writing Group Members, Mozaffarian, D., Benjamin, E. J., Go, A. S., Arnett, D. K., Blaha, M. J., Cushman, M., Das, S. R., de Ferranti, S., Després, J.-P., 
Fullerton, H. J., Howard, V. J., Huffman, M. D., Isasi, C. R., Jiménez, M. C., Judd, S. E., Kissela, B. M., Lichtman, J. H., Lisabeth, L. D., ... Stroke Statistics Subcommittee. (2016). Heart Disease and Stroke Statistics-2016 Update: A Report From the American Heart Association. Circulation, 133(4), e38e360.

Xia, Y., Zhu, K., Lai, H., Lang, M., Xiao, Y., Lian, S., Guo, C., \& Wang, C. (2015). Enhanced infarct myocardium repair mediated by thermosensitive copolymer hydrogel-based stem cell transplantation. Experimental Biology and Medicine , 240(5), 593-600.

Yancy, C. W., Jessup, M., Bozkurt, B., Butler, J., Casey, D. E., Jr, Colvin, M. M., Drazner, M. H., Filippatos, G. S., Fonarow, G. C., Givertz, M. M., Hollenberg, S. M., Lindenfeld, J., Masoudi, F. A., McBride, P. E., Peterson, P. N., Stevenson, L. W., \& Westlake, C. (2017). 2017 ACC/AHA/HFSA Focused Update of the 2013 ACCF/AHA Guideline for the Management of Heart Failure: A Report of the American College of Cardiology/American Heart Association Task Force on Clinical Practice Guidelines and the Heart Failure Society of America. Journal of Cardiac Failure, 23(8), 628-651.

Zhu, Y., Wood, N. A., Fok, K., Yoshizumi, T., Park, D. W., Jiang, H., Schwartzman, D. S., Zenati, M. A., Uchibori, T., Wagner, W. R., \& Riviere, C. N. (2016). Design of a Coupled Thermoresponsive Hydrogel and Robotic System for Postinfarct Biomaterial Injection Therapy. The Annals of Thoracic Surgery, 102(3), 780-786. 